\title{
MULTI-MODE SWITCHING CONTROL OF HYBRID ELECTROMAGNETIC SUSPENSION BASED ON ROAD CONDITIONS ADAPTATION
}

\author{
Xiangpeng Meng, Renkai Ding, Zeyu Sun, Ruochen Wang, Long Chen \\ School of Automobile and Traffic Engineering, Jiangsu University, Zhenjiang, China \\ e-mail: mengxp@ujs.edu.cn; dingrk0929@sina.com; szy060@163.com; wrc@ujs.edu.cn; chenlong@ujs.edu.cn
}

\begin{abstract}
In this research, a hybrid electromagnetic actuator is proposed to coordinate the contradictions between dynamic performance and energy consumption of an electromagnetic suspension. The hybrid electromagnetic suspension (HEMS) is configured to operate in the passive energy regeneration mode, active control ride comfort mode or active control driving safety mode depending on the road excitation frequency. Then, the HEMS system is modeled. The simulation results show that the HEMS can automatically switch between different modes, and realize an effective coordination between dynamic performance and energy saving. Finally, a quarter car test is conducted, which verifies the effectiveness of the multi-mode switching control.
\end{abstract}

Keywords: hybrid electromagnetic suspension, multi-mode switching control, road conditions adaptation, dynamic performance, energy regeneration

\section{Introduction}

Energy-saving is the main direction of the current automotive industry, especially for electric vehicles, as energy-saving technology can further improve its endurance mileage (Ding et al., 2019). The most obvious case is the regenerative braking technology (Lv et al., 2015). Compared to the relatively mature regenerative braking (which has been applied in new energy vehicles such as Toyota Prius), another energy-saving technology: vibration energy regeneration has also attracted attention of many scholars (Ding et al., 2017, 2019; Xie and Wang, 2015). The main purpose of the technology is to convert vibration energy generated by the suspension system into electrical energy for reuse.

In order to reuse vibration energy, Suda integrated the rotating motor into the suspension system to convert linear reciprocating motion of the body and the wheel into rotation of motor through the ball screw structure (Suda et al., 2004). Based on the same structure, Li et al. (2013) fully evaluated regenerative vibration energy of the suspension system, analyzed the influence of structural parameters on the regeneration power. In the energy regeneration process, the motor is used as a generator whether it is a rotating or linear one, then the suspension system works as a PS, and the dynamic performance of the suspension system (Ride comfort, driving safety, etc.) will inevitably be affected by the regeneration process (Ataei et al., 2017). Therefore, how to effectively coordinate the contradictory relationship between the regeneration power and dynamic performance remains the key problem in vibration energy regeneration technology.

In the regenerative braking technique, the rotary motor is used to drive the vehicle when it is operated in the motor mode, while in the suspension system, the rotary motor/linear motor is mainly used for active control to improve the dynamic performance of the system when it works as an electric motor. And the linear motor is an ideal alternative to the traditional hydraulic damper because of its simple structure, easy setting, and no requirement of intermediate transmission mechanism. Martins used a linear motor as the suspension system actuator, designed 
its structure and parameters, and verified the feasibility of the program through experiments (Martins et al., 2006). Wang took high thrust density as the design goal to optimize the structure, and designed a linear motor that meets the requirements of the suspension system (Wang et al., 2011). Tang optimized the structure and parameters of a linear motor and improved the regeneration power (Zuo et al., 2011). The above studies demonstrate that the application of the linear motor in an active control of suspension system is feasible, but it should be noted that the additional energy consumption limits its use in the vehicles. In summary, the dynamic performance of the suspension system will be affected when using the linear motor to regenerate vibration energy, while energy consumption is the main factor that limits its application when applying active control. In general, energy saving and good dynamic performance cannot be achieved at the same time. In order to coordinate the two sides effectively, scholars have given the following solutions. Solution one: semi-active control. Specifically, Asadi and Ribeiro integrated the linear motor with the hydraulic damper to form a HEMS in which the base damping was provided with the hydraulic damper to change the external resistance during the energy regeneration process of the linear motor, thereby making the overall damping value adjustable. Energy saving and the dynamic performance of suspension system were considered in the process (Asadi et al., 2017). Shi et al. (2014) connected a linear motor and a hydraulic adjustable damper in parallel. Vibration energy was only regenerated by the linear motor, and the damping value could be adjusted within three levels, so as to meet the requirements of dynamic performance under different driving conditions. Solution two: mode switch between active control and energy regeneration. David used a linear motor as the actuator and realized passive, semi-active and active switching of the suspension system by designing the control circuit and the dynamic controller (Bose suspension system, 2004), but the switching needs to be done manually by the driver.

The above research results can effectively coordinate energy saving and dynamic performance, but it still has the following questions during the mode switching between active control and energy regeneration: On the one hand, the function of a linear motor is equivalent to an electromagnetic damper during the process of energy regeneration, and its damping force is much smaller than that of the passive damper. So, the effect of rapid attenuation of vibration of the hydraulic damper cannot be achieved. On the other hand, a single linear motor structure cannot guarantee reliability of the suspension system, and the system cannot work properly when the power supply fails. In view of the above problems, this paper presents an integrated hybrid electromagnetic damper/actuator to conduct a research on multi-mode switching control of HEMS. Based on different road conditions, the working mode of the suspension system is divided into the passive energy regeneration mode, active control comfort mode and active control security mode (Wang et al., 2018; Sun et al., 2017a,b). The switching threshold is set, and a new multi-mode switching energy based on road conditions adaption is proposed to achieve effective coordination between energy saving and dynamic performance.

The main work and innovations of this paper are as follows: integrate the traditional hydraulic damper with the linear motor, and design the hybrid electromagnetic damper/actuator as the implementing mechanism of the suspension system. On this basis, according to the reversibility principle of the linear motor, it determines a variety of working modes of HEMS (including passive regeneration mode, active control comfort mode, and active control safety mode) and the switching thresholds which can be automatically switched among various working modes according to various road conditions.

\section{Hybrid electromagnetic actuator}

The biggest problem of applying a linear motor instead of the traditional hydraulic damper for vibration energy regeneration or active control is reliability of the system (Martins et al., 
1999). As shown in Fig. 1, it integrates a linear motor with hydraulic dampers to form a hybrid electromagnetic damper. The hydraulic damper is used as the reference design and the center pole of the linear motor.

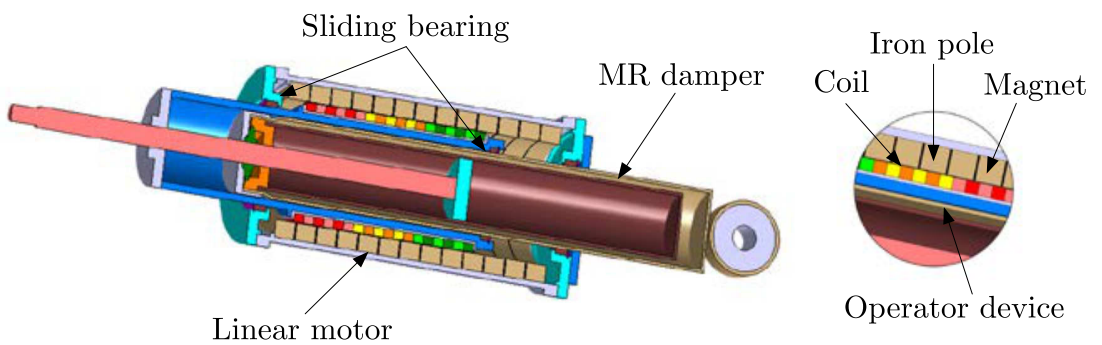

Fig. 1. Integrated hybrid electromagnetic actuator

Taking the $1 / 4$ of a linear suspension system as the research object, the linear dynamic model of two degrees of freedom is established (Fig. 2a). According to the model, the kinetic equation can be expressed as follows

$$
\begin{aligned}
& m_{s} \ddot{z}_{s}=-k_{s}\left(z_{s}-z_{u}\right)-c\left(\dot{z}_{s}-\dot{z}_{u}\right)+F_{h} \\
& m_{u} \ddot{z}_{u}=k_{s}\left(z_{s}-z_{u}\right)+c\left(\dot{z}_{s}-\dot{z}_{u}\right)-k_{t}\left(z_{u}-z_{r}\right)-F_{h}
\end{aligned}
$$

where $m_{s}$ is the sprung mass, $m_{u}$ is the unsprung mass, $k_{s}, k_{t}, c$ is spring stiffness, tire stiffness and hydraulic damping coefficient, respectively, $z_{s}, z_{u}, z_{r}$ is body, wheel and road displacement. $F_{h}$ is the force produced by the linear motor.

(a)

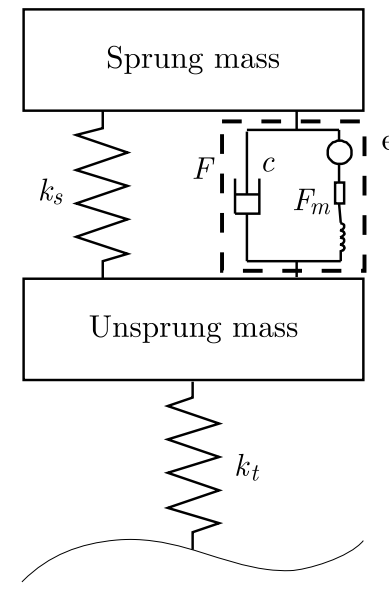

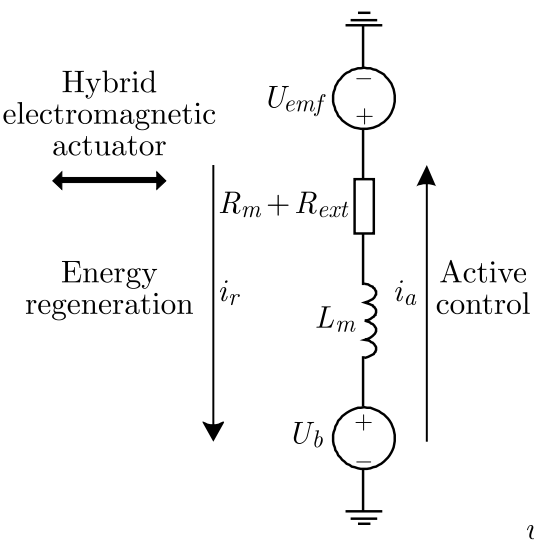

(b)

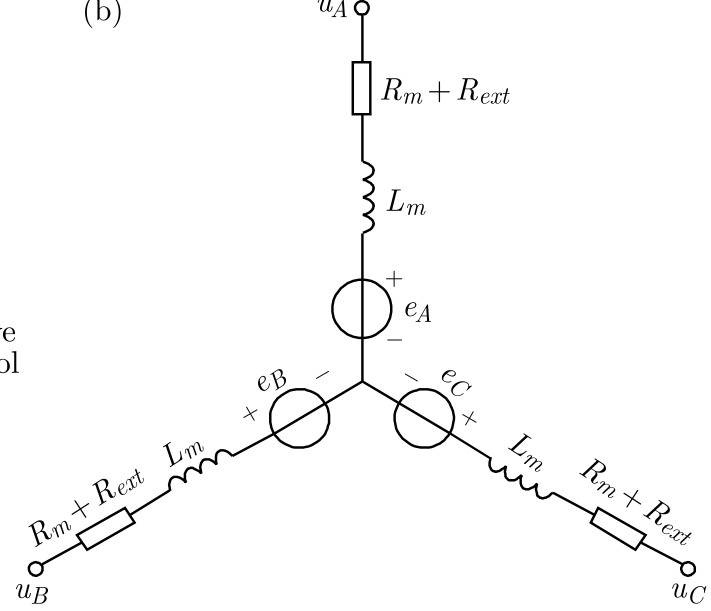

Fig. 2. Model of the HEMS system: (a) dynamic model, (b) equivalent circuit of linear motor

According to the working mode of the linear motor, $F_{h}$ can be expressed as

$$
F_{h}= \begin{cases}\frac{k_{e}}{R_{m}+R_{e x t}} U_{e m f}=k_{e} i_{r} & \text { energy regeneration } \\ \frac{k_{i}}{R_{m}+R_{e x t}}\left(U_{b}-U_{e m f}\right)=k_{i} i_{a} & \text { active control }\end{cases}
$$

where $U_{\text {emf }}$ is the back electromotive force (EMF) produced by the linear motor, and $U_{b}$ is the supply voltage of the battery. As shown in Fig. 2b, if three-phase windings of linear motor are star-connected and symmetrical in space, assuming that the magnetic circuit is unsaturated, regardless of eddy current and hysteresis losses, the resistance, self-inductance and mutual inductance of all stator windings are equal and constant, there is a voltage equation for each 
winding phase in the absence of midline lead-out in the energy regeneration mode and active control mode, which can be expressed as

$$
U_{e m f}=R_{m} i_{r}+L_{m} \frac{d i_{r}}{d t}+R_{e x t} i_{r} \quad U_{b}=\left(R_{m}+R_{e x t}\right) i_{a}+L_{m} \frac{d i_{a}}{d t}+U_{e m f}
$$

where $k_{e}$ and $k_{i}$ are the back EMF coefficient and the thrust coefficient of the linear motor, respectively. $R_{m}$ is internal resistance; $R_{e x t}$ is external resistance which is to be adjusted to the regeneration power (when the external resistance equals to the internal resistance, the maximum power can be obtained, thus, the same values of $R_{m}$ and $R_{\text {ext }}$ are selected) (Wang et al., 2001). $L_{m}$ is motor inductance. Considering that the vibration frequency of the vehicle caused by the road displacement input is relatively small $(0.5-25 \mathrm{~Hz})$ and the motor inductance is relatively small, so the effects of the motor inductance are ignored in this paper.

At the same time, the average power of regeneration/consumption of the linear motor in different working modes can be expressed as follows

$$
\bar{P}= \begin{cases}\frac{1}{t} \int_{0}^{t} U_{e m f} i_{r} d t & \text { energy regeneration } \\ \frac{1}{t} \int_{0}^{t} U_{b} i_{a} d t & \text { active control }\end{cases}
$$

Taking the wave-filtered white noise as the road surface input, it can be expressed as follows

$$
\dot{z}_{r}=-2 \pi f_{0} \dot{z}_{r}+2 \pi n_{0} \sqrt{G_{q}\left(n_{0}\right) v} w
$$

The system parameters are shown in Table 1.

Table 1. Parameters of the HEMS system

\begin{tabular}{|c|c|c|c|c|c|}
\hline Parameter & Value & Description & Parameter & Value & Description \\
\hline \hline$m_{s}$ & $210 \mathrm{~kg}$ & Sprung mass & $k_{i}$ & $52.6 \mathrm{~N} / \mathrm{A}$ & Thrust coefficient \\
\hline$m_{u}$ & $25 \mathrm{~kg}$ & Unsprung mass & $k_{e}$ & $42.9 \mathrm{Vs} / \mathrm{m}$ & EMF coefficient \\
\hline$k_{s}$ & $12 \mathrm{kN} / \mathrm{m}$ & Spring stiffness & $R_{m}$ & $1.69 \Omega$ & Motor resistance \\
\hline$k_{t}$ & $120 \mathrm{kN} / \mathrm{m}$ & Tire stiffness & $R_{e x t}$ & $1.69 \Omega$ & External resistance \\
\hline$c$ & $1000 \mathrm{Ns} / \mathrm{m}$ & Damping coefficient & $L_{m}$ & $2.59 \mathrm{mH}$ & Motor inductance \\
\hline$f_{0}$ & $0.1 \mathrm{~Hz}$ & Cutoff frequency & $n_{0}$ & $0.1 \mathrm{~m}^{-1}$ & Spatial frequency \\
\hline$G_{q}\left(n_{0}\right)$ & $256 \mathrm{e}-6 \mathrm{~m}^{-3}$ & Road roughness coeff. & $v$ & $20 \mathrm{~m} / \mathrm{s}$ & Vehicle speed \\
\hline
\end{tabular}

\section{Multi-mode switching strategy}

It is well known that the regeneration power is influenced by the input of road surface displacement and the vehicle speed when the linear motor is used to regenerate the vibration energy. Vehicle speed can also be understood as the road excitation frequency $\left(f=u^{*} n, f, u, n\right.$ are time frequency, speed and spatial frequency, respectively), and the faster the speed, the higher the excitation frequency, so the regeneration power is mainly related to driving conditions. In other words, under the same excitation frequency, if the road displacement input is greater, the relative velocity between the body and the wheel will inevitably be greater, which will lead to greater regeneration power. However, under the same road displacement input, the higher excitation frequency does not mean the greater regeneration power. It can be seen from Fig. 3 that 
the relative velocity peak of the body and the wheel appears at the resonance frequency of the vehicle body and the wheel respectively under the excitation of the road surface. Thus, under the same road surface displacement input, the regeneration power is improved by the body and wheel resonance zone, especially the wheel resonance zone.

However, from the perspective of vehicle dynamic performance, the body resonance will greatly reduce the ride comfort, and wheel resonance will affect the wheel grounding, that is, reduce driving safety. Therefore, how to effectively suppress the adverse effects of the body-wheel resonance is a problem the scholars concern the most. Generally speaking, the frequency range of the body resonance is $0.5 \mathrm{~Hz}-4 \mathrm{~Hz}$, and the frequency range of the wheel resonance is $8 \mathrm{~Hz}-12 \mathrm{~Hz}$. In this frequency domain, the anti-resonance between the body and the wheel can be realized if the ceiling, and the floor shed is used respectively. The dynamic performance can be improved effectively.

Table 2. Driving speed under different road situations

\begin{tabular}{|c|c|c|}
\hline Driving situation & Road condition & Driving speed $[\mathrm{Km} / \mathrm{h}]$ \\
\hline \hline Highway I & Grade-A & 120 \\
\hline Highway II & Grade-B & 90 \\
\hline City & Grade-C & 50 \\
\hline Off-road & Grade-D & 30 \\
\hline
\end{tabular}

Based on the above analysis and the reversibility principle of the linear motor, the working modes of HEMS are divided into three types: passive energy regeneration mode, active control comfort mode and active control safety mode. Taking the road excitation frequency as the switching threshold of each mode, the energy-saving and dynamic performance of the suspension system are all taken into account through the automatic switching of each mode. It should be noted that the road excitation frequency always depends on the road displacement input. As shown in Table 2, the smaller the input of road displacement, the faster the vehicle speed, and the higher the road excitation frequency. However, according to the input of road displacement, it is impossible to effectively determine the switching time between the active and passive mode. Therefore, the road excitation frequency is taken as the switching threshold, while defining the working frequency band of the active mode, and the remaining frequency band is set as the passive mode so as to achieve the purpose of automatic switching. The working frequency bands of each mode and their corresponding control strategies are shown in Table 3. To avoid the "dead zone" in the passive energy regeneration mode, a DC/DC boost converter is integrated to adjust the charging voltage.

Table 3. Control targets and control methods under different frequency methods

\begin{tabular}{|c|c|c|c|}
\hline Frequency band $[\mathrm{Hz}]$ & Working mode & Control object & Control strategy \\
\hline \hline $0.5-4$ & Active & Ride comfort & Skyhook control \\
\hline $4-8 \& 12-25$ & Energy regeneration & Energy regeneration & DC-DC boost \\
\hline $8-12$ & Active & Road holding & Ground-hook control \\
\hline
\end{tabular}

\section{Identification methods for identification of road excitation frequency}

Section 3 sets the road excitation frequency as the threshold of each mode switching. Therefore, identifying the road excitation frequency is the basis for realizing the automatic switching in each mode. In this paper, the first-order zero-crossing detection algorithm is used to identify the road excitation frequency (Wang et al., 2001) by estimating the input of the road speed, but the 
feasibility of the algorithm is not verified. In fact, it is difficult to measure or estimate the road speed input in real-time and accurately during the vehicle normal running process. However, the frequency response characteristic of the suspension relative velocity under the input of road speed can be expressed as follows

$$
H(j w)_{\left(\dot{z}_{s}-\dot{z}_{u}\right) \sim \dot{z}_{r}}=\frac{\dot{z}_{s}-\dot{z}_{u}}{\dot{z}_{r}} \mathrm{e}^{\mathrm{j}\left(\varphi_{2}-\varphi_{1}\right)}
$$

It can be seen from Eq. (4.1) that the response frequency of the relative velocity of the suspension is consistent with the input frequency of the road speed, but there is a response delay (phase difference). Thus, in this paper, the first-order zero-crossing detection algorithm is applied to identify the response frequency of the relative velocity of the suspension, so as to indirectly obtain the road excitation frequency.

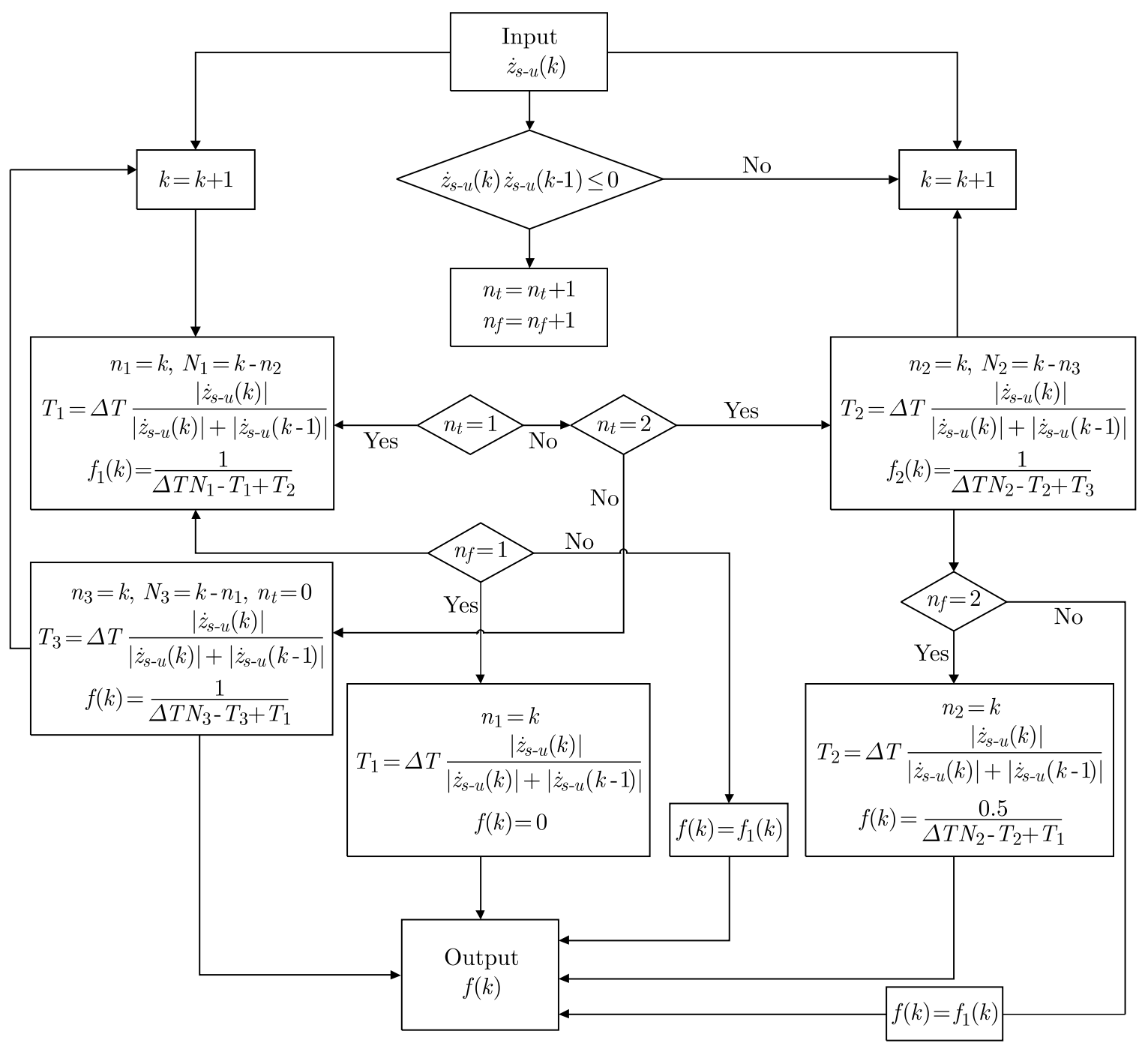

Fig. 3. Flow chart of the first-order zero-crossing detection algorithm

The flow chart of the first-order zero-crossing detection algorithm is shown in Fig. 3. The relative velocity passing through zero for three consecutive times is assumed as one cycle, and the product of the relative velocity of two consecutive single-steps sampling times (sample $k-1$ and sample $k$ ) is detected. If the product is negative or zero, there is at least one zero point between the two sampling points. In order to calculate the time interval between the first zero point and the third zero point more accurately, linear interpolation is used in the interval between 
the zero point and its previous sampling point. In the figure, $T_{1}, T_{2}$ and $T_{3}$ represent the time interval between each zero point and its previous sampling point respectively; $n_{1}, n_{2}$ and $n_{3}$ are the intermediate parameters, which are respectively used to record the sampling numbers of the three zero points during the sampling process; $N_{1}, N_{2}$ and $N_{3}$ are the sampling pitches between the two non-adjacent zero points. $f(k)$ is the identification result of the response frequency of the suspension relative velocity at the $k$-th sampling point.

\section{Simulation analysis}

\subsection{Control system design}

As shown in Fig. 4, a double-loop control system is designed. The outer loop is the suspension control system, in which the acceleration of the body and wheel and the relative displacement of the body and the wheel are measured by using acceleration sensors and displacement sensors. The relative displacement is differentiated to obtain the relative velocity, so that the road excitation frequency is achieved indirectly according to the first-order zero-crossing detection algorithm proposed in Section 4. Then the working mode of the suspension system (linear motor) can be judged according to the road excitation frequency. If it works in the active mode, an ideal control force is output to the inner loop by the controller. If it works in the passive regeneration mode, no control output is available. The ideal control force with the skyhook $(0.5 \mathrm{~Hz}-4 \mathrm{~Hz}) /$ groundhook $(8 \mathrm{~Hz}-12 \mathrm{~Hz})$ control strategy for the active control mode can be expressed as follows

$$
F_{\text {des }}= \begin{cases}-c_{s} \dot{z}_{s} & \text { skyhook control } \\ -c_{g} \dot{z}_{u} & \text { groundhook control }\end{cases}
$$

And the actual active force is shown in equation (2.2), thus, the supply current $i_{a}$ can be obtained according to equations (2.2) and (5.1).

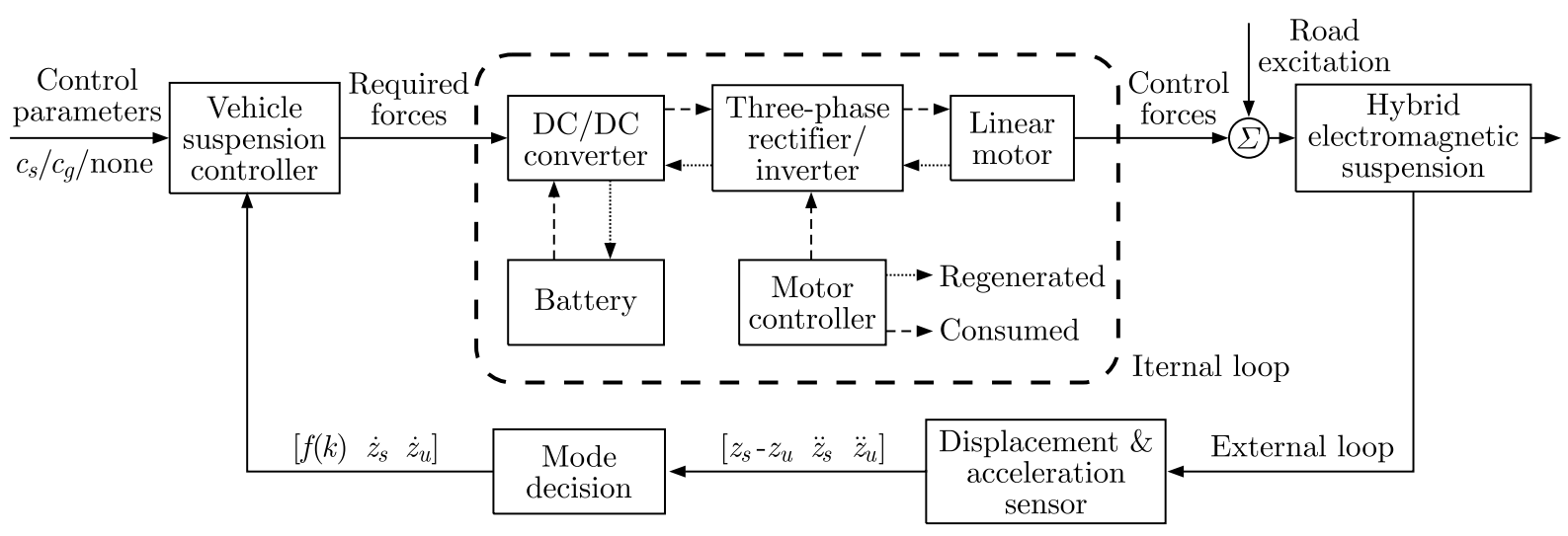

Fig. 4. Control system

\subsection{Dynamical analysis of frequency domain}

In the active control mode, selection of the skyhook damping coefficient $c_{s}$ and ground hook damping coefficient $c_{g}$ has an important effect on the control efficiency. As shown in Fig. 5, the gain of the vehicle acceleration decreases as the skyhook damping coefficient increases in the vehicle body resonance zone, which makes people feel more comfortable. Given the same situation, the driving safety enhances as the ground hook damping coefficient increases in the tire resonance area. However, increasing the skyhook/ground hook damping coefficient increases 

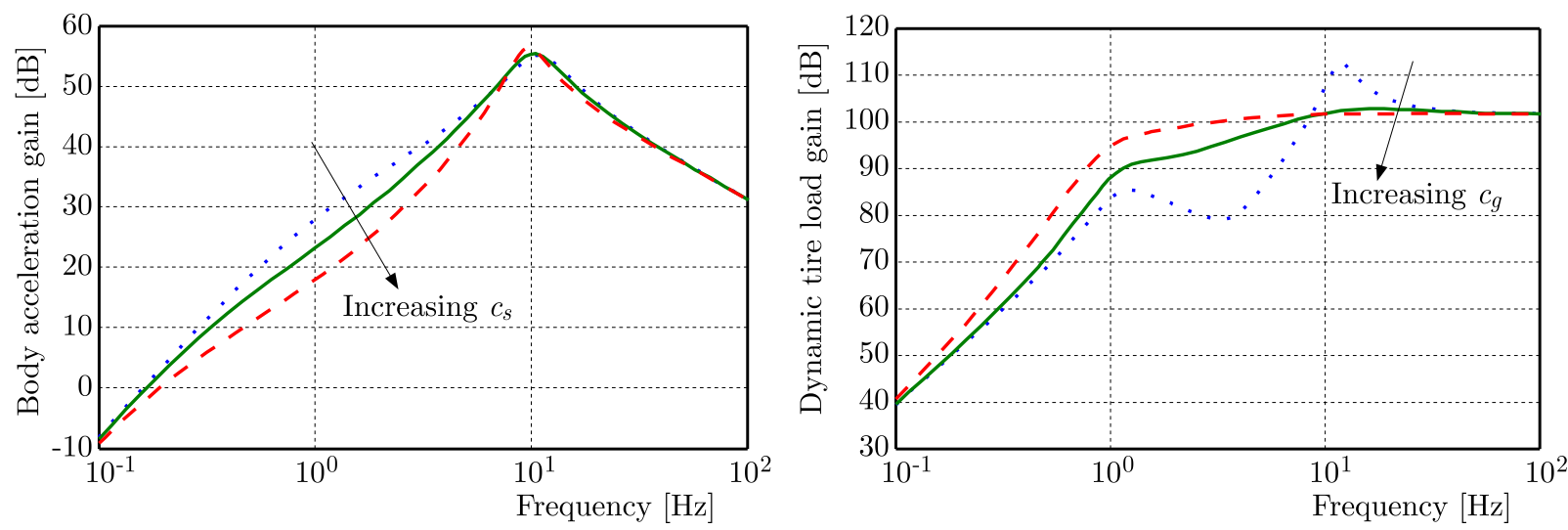

Fig. 5. Influence of control parameters on control efficiency

the ideal control force in the same driving road conditions. That is, the actual output force generated by the linear motor will increase.

Thus, because it is limited to the maximum output force generated by the linear motor, the skyhook and ground hook damping coefficients selected in this paper are $c_{s}=2730 \mathrm{Ns} / \mathrm{m}$, $c_{g}=1450 \mathrm{Ns} / \mathrm{m}$.

The PS is selected as the object of comparison to study the dynamic performance of HEMS under different working modes (different frequency bands) respectively. As shown in Fig. 6, the gain of the body acceleration is effectively reduced by using the skyhook control strategy in the body resonance zone, that is, it can improve ride comfort ability. At the same time, the gain of the tire dynamic load of this band is also reduced, it means that the driving safety has been greatly improved. In the wheel resonance zone (Fig. 7), the driving safety is improved because of the use of the ground hook control strategy to suppress the wheel resonance.
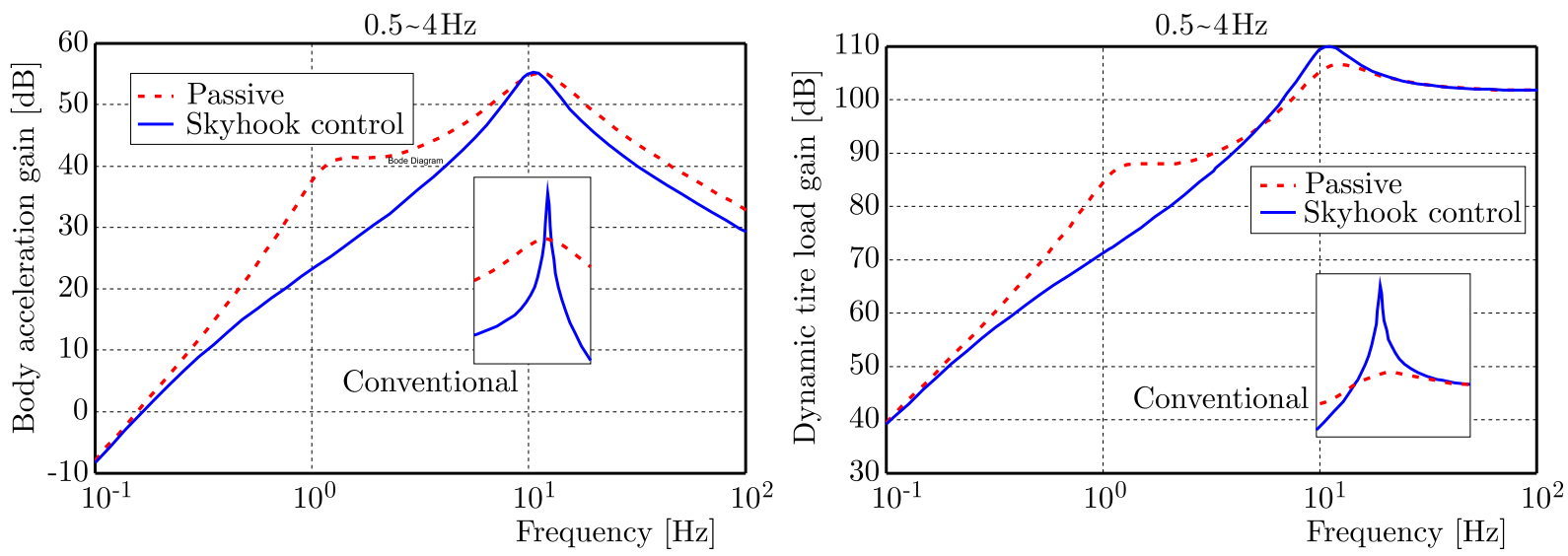

Fig. 6. Dynamic performance comparisons between HEMS under the skyhook control and traditional suspension

The above results show that the dynamic performance in the corresponding band can be taken into consideration respectively by adopting the skyhook/ground hook control strategy. As shown in Fig. 8, the gain of both the body acceleration and the dynamic tire load are increased in the $4 \mathrm{~Hz}-8 \mathrm{~Hz}$ band, but the outcome is opposite in the $12 \mathrm{~Hz}-25 \mathrm{~Hz}$ band. This is mainly because of the integration of the hydraulic damper with the linear motor, which increases the sprung and unsprung mass, so it causes a change in the ratio of the sprung to unsprung mass. However, due to the optimized design of the hydraulic damper structure, the variation amplitude of the mass ratio is relatively small (Table 1), so the dynamic performance of the suspension system does not deteriorate greatly in the process of energy regeneration. 

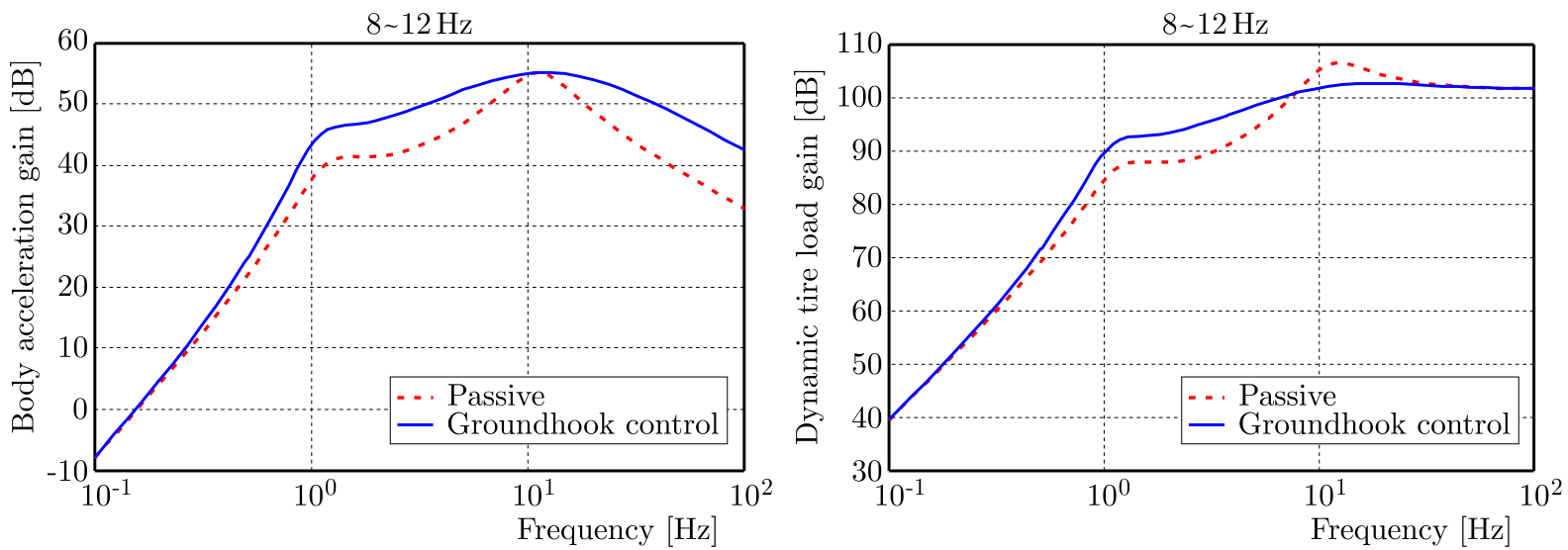

Fig. 7. Dynamic performance comparisons between HEMS under the groundhook control and traditional suspension
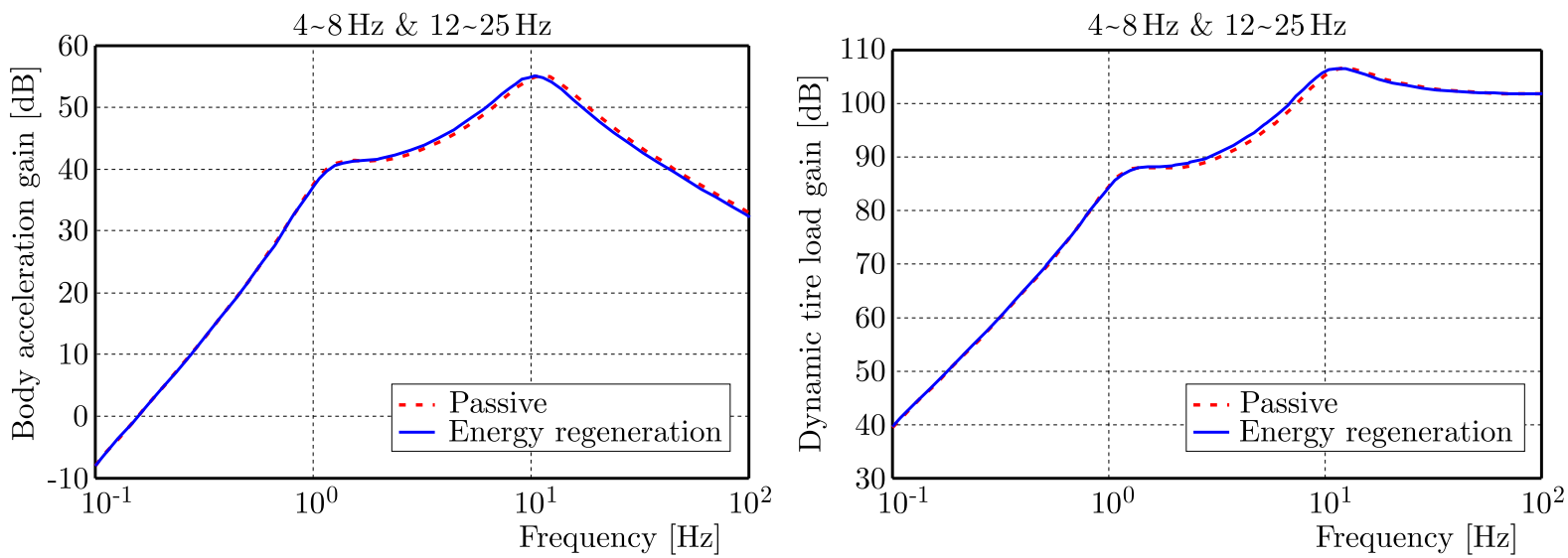

Fig. 8. Dynamic performance comparisons between HEMS under the energy regeneration mode and traditional suspension

According to the above analyses, compared with the PS, the HEMS can achieve its corresponding control objectives under different working modes. Specifically, riding comfort and driving safety of the corresponding band can be improved in the active control mode. In the passive regeneration mode, energy regeneration can be achieved without deterioration of the dynamic performance of the system. It can be seen that the control strategy in different control modes is effective, and the design of the hybrid electromagnetic actuator is rational.

\subsection{Analysis of dynamics and energy consumption in time domain}

This Section will verify feasibility of the identification algorithm on the road excitation frequency and the effectiveness of the control system by using time domain simulation analysis. As shown in Fig. 9a, the sinusoidal input is used in the road excitation. And in Table 3, four working conditions are set correspondingly according to the working frequency bands of the HEMS in different modes. In order to simulate the actual situation of the vehicle running process, the relationship between the input displacement and the excitation frequency should be taken into account. This means that the greater the input displacement, the smaller the excitation frequency. Figure 9b shows the results of indirect identification on the road excitation frequency by using the first-order zero-crossing detection algorithm. It can be seen from the figure that the road excitation frequency can be accurately identified with the algorithm, which provides the necessary condition for realizing the automatic switching between different modes. 
(a)

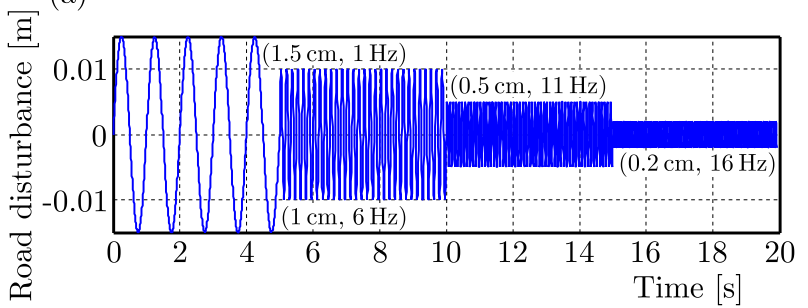

(b)

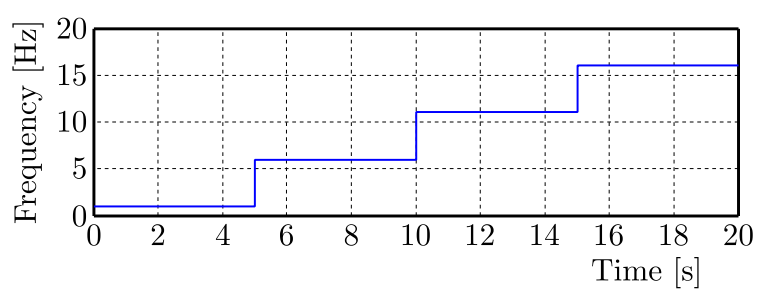

Fig. 9. (a) Road surface input, (b) frequency detection
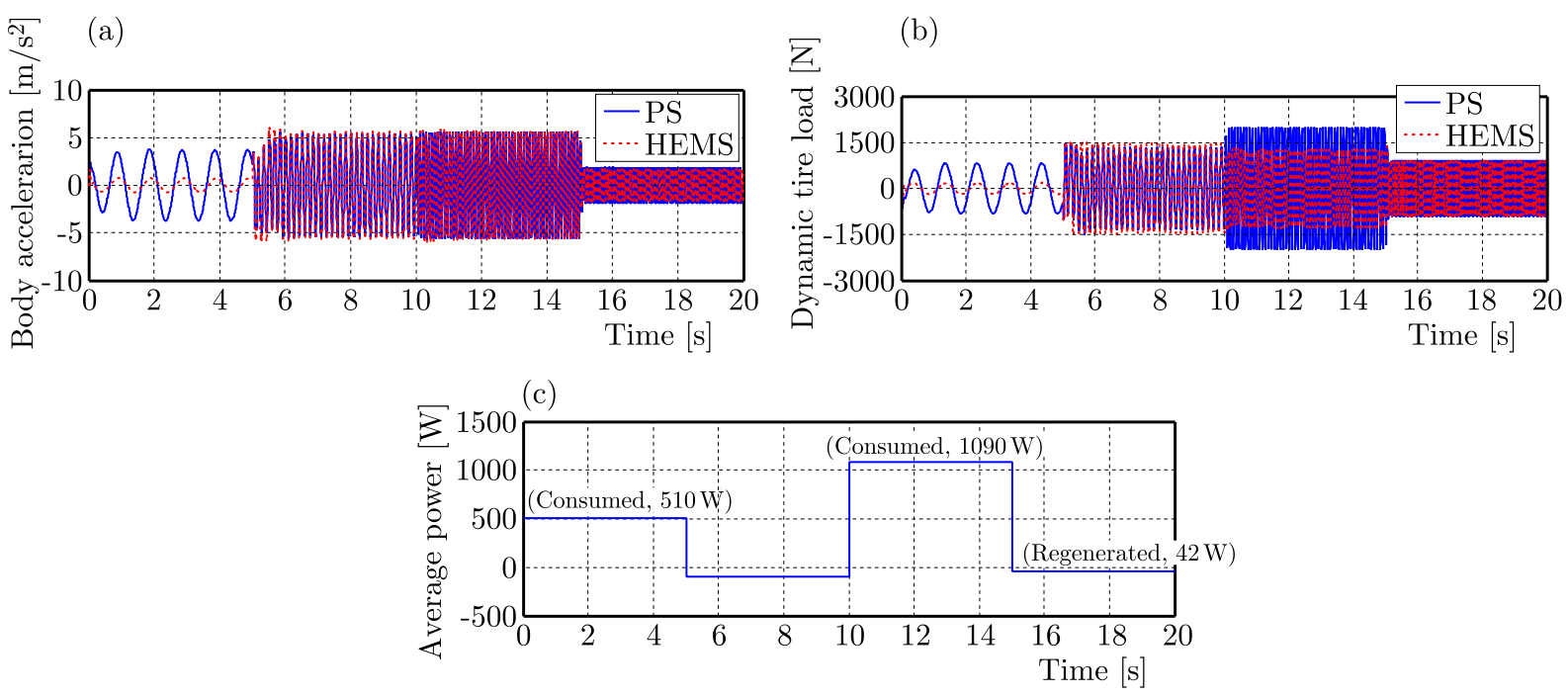

Fig. 10. Simulation results: (a) body acceleration, (b) dynamic tire load, (c) average consumed/regenerated power

Figure 10 shows the body acceleration, tire dynamic load and energy consumption/regeneration power of the HEMS under the road excitation. Compared with the PS, from Fig. 10a and 10b, it can be seen that the body acceleration and the tire dynamic load of the HEMS are significantly reduced in the active control comfort mode. More specifically (Table 4), the body acceleration is reduced by $78 \%$ and the RMS value of wheel dynamic load is reduced by $77.2 \%$. In the active control safety mode, the tire dynamic load of the HEMS is significantly reduced (by $22.2 \%$ ), but the body acceleration does not increase. The results are consistent with that of Section 6.1, which shows that the dynamic performance in the corresponding bands can be respectively taken into account by adopting the skyhook/ground hook control strategy based on the HEMS. When it works in the passive regeneration mode, the body acceleration and the dynamic tire load are slightly increased in the $4 \mathrm{~Hz}-8 \mathrm{~Hz}$ band, but there is an opposite result in the $12 \mathrm{~Hz}-25 \mathrm{~Hz}$ band. The above results reveal that the control system designed in this paper can realize excellent control of the HEMS under different working modes according to the control strategy, which verifies the effectiveness of the control system.

Table 4. Comparison of dynamic performance under different modes

\begin{tabular}{|l|c|c|c|c|c|}
\hline \multicolumn{2}{|c|}{$\begin{array}{c}\text { Performance index } \\
(\text { Peak-to-peak })\end{array}$} & $\begin{array}{c}\text { Ride comfort } \\
(0.5 \mathrm{~Hz}-4 \mathrm{~Hz})\end{array}$ & $\begin{array}{c}\text { Energy regenerat. } \\
(4 \mathrm{~Hz}-8 \mathrm{~Hz})\end{array}$ & $\begin{array}{c}\text { Road holding } \\
(8 \mathrm{~Hz}-12 \mathrm{~Hz})\end{array}$ & $\begin{array}{c}\text { Energy regenerat. } \\
(12 \mathrm{~Hz}-25 \mathrm{~Hz})\end{array}$ \\
\hline \hline \multirow{2}{*}{$\begin{array}{l}\text { Body accele- } \\
\text { ration }\left[\mathrm{m} / \mathrm{s}^{2}\right]\end{array}$} & HEMS & 2.04 & 10.85 & 10.84 & 2.52 \\
\hline \multirow{2}{*}{$\begin{array}{l}\text { Dynamic tire } \\
\text { load }[\mathrm{N}]\end{array}$} & HEMS & 9.28 & 10.65 & 10.92 & 2.54 \\
\cline { 2 - 6 } & PS & 2337.8 & 3027.9 & 2634 & 2385.8 \\
\hline
\end{tabular}


Figure 10c shows the average power of consumption/regeneration of the HEMS under different working modes. It can be seen from the figure:

- Power consumption in the active control safety mode is significantly greater than that in the comfort mode. On one hand, this is because the safety mode requires "hard damping" while the comfort mode requires "soft damping", which results in the output force of the linear motor in the safety mode greater than that in the comfort mode. On the other hand, the frequency band of the security mode is higher than that of the comfort mode, which means the response frequency of the linear motor in the safety mode is higher than that in the comfort mode.

- The regenerated power in the passive energy regeneration mode accounted for $8.75 \%$ of the consumed power in the active control mode. That is, the regenerated power can be used to compensate for the consumed power in the active control mode, so as to achieve the purpose of energy saving.

In conclusion, the correctness of the identification algorithm on the road excitation frequency, the effectiveness of control strategy and control system under different modes, and the feasibility of energy saving realized by multi-mode switching were verified by simulation analysis.

\section{Bench test}

\subsection{Structure arrangement}

Now verify feasibility of the multi-mode switching control strategy based on adaption of road conditions in the HEMS. In other words, in order to verify correctness of the simulation results, a two-degree-of-freedom test bench is constructed. As shown in Fig. 11, the input is simulated by the INSTRON 8800 CNC hydraulic choke (consistent with simulation). Six sets of springs

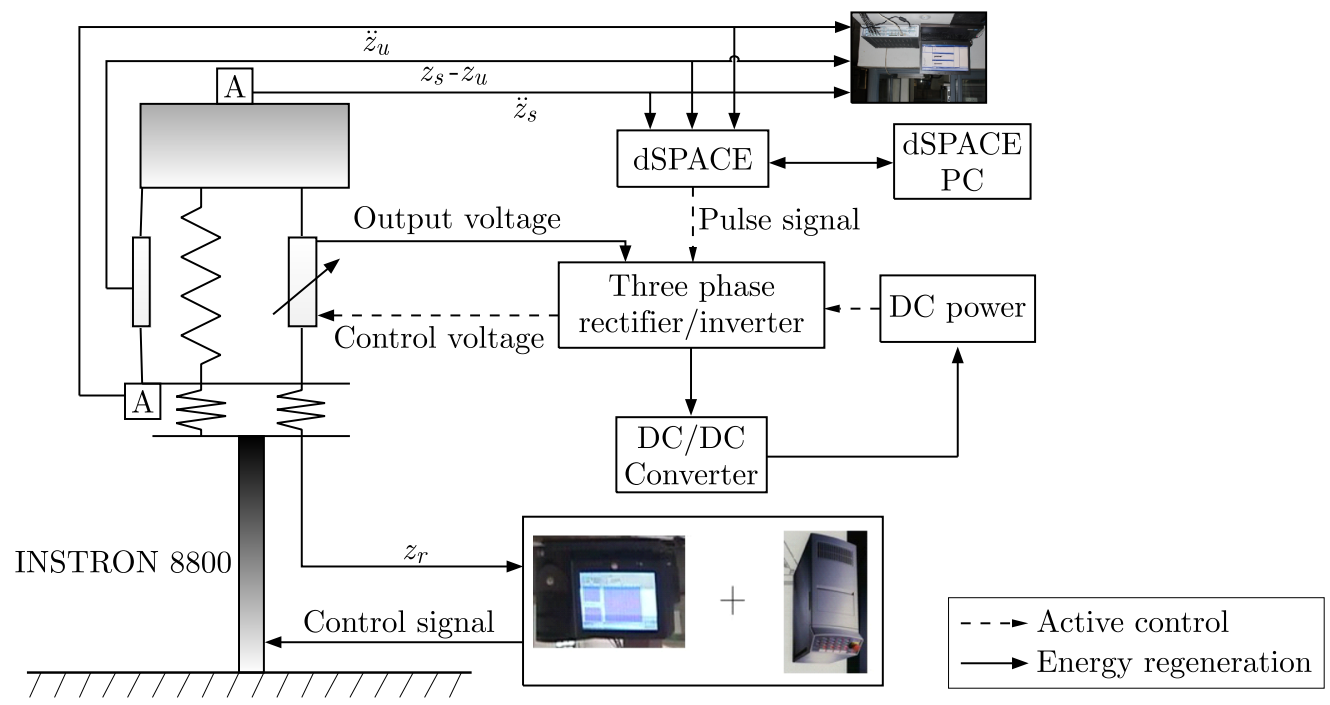

Fig. 11. Bench test configuration

are used to simulate tire stiffness, acceleration and displacement sensors are used to measure the acceleration and relative displacement of the body and the wheel, respectively. On one hand, the measured data is the input to the LMS data acquisition instrument, on the other, it is the input to the controller based on the dSPACE rapid prototyping. Unlike the data measured by the LMS acquisition, the controller needs to be filtered to eliminate the interference of the system and measurement noise (LMS data can be filtered off-line) before receiving the data. And as for the sky/ground hook control strategy, the acceleration needs to be integrated, so as to provide the absolute speed of the body and wheel. The implementation process of multi-mode switching 
is as follows: According to the input signal provided by the displacement sensor, the dynamic response frequency of the suspension is calculated in real time to control the working mode of the linear motor, so as to complete the energy conversion between the DC power supply and the motor (energy supply/ energy regeneration). It should be noted that when the linear motor operates in the motor mode, its maximum back electromotive force is always lower than the supply voltage. Therefore, when the linear motor operates in the generator mode, it needs to integrate the DC boost converter to improve its charging voltage. During the test, the terminal voltage and its energy supply/regeneration current of the DC power supply are measured by the voltage sensor and the current sensor, respectively.

\subsection{Experimental results}

After off-line filtering of the sensor data collected by LMS, the body acceleration and the dynamic wheel load are obtained as shown in Fig.13. The tire stiffness and road input are given, so the dynamic tire load can be acquired through calculating the wheel displacement which is obtained by quadratic integration of the wheel acceleration. It can be seen from the figure that the HEMS can realize working mode switching in different frequency bands, but there is a time lag in the switching process, and the system also produces a certain degree of jitter. Therefore, how to improve the response time of the system switching and the stability in the handover process will remain as the focus of the follow-up work (no discussion here).
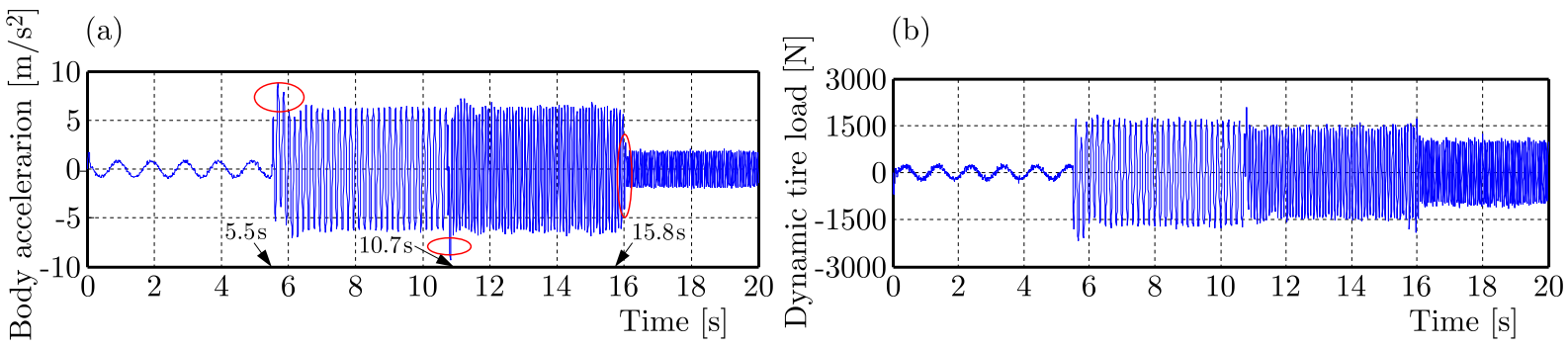

Fig. 12. Test results: (a) body acceleration, (b) dynamic tire load

(a)

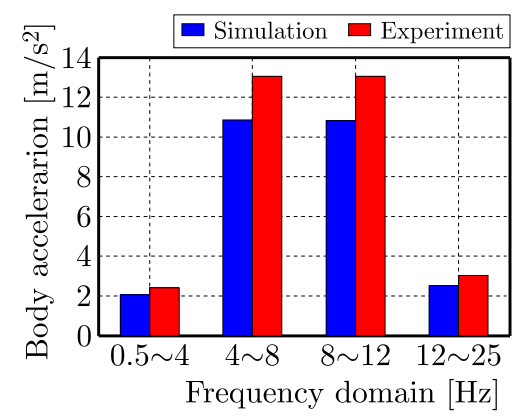

(b)

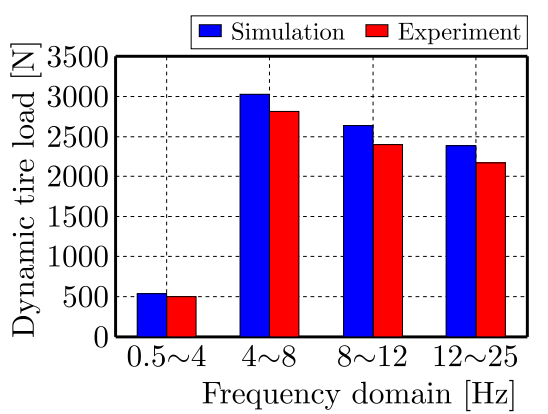

(c)

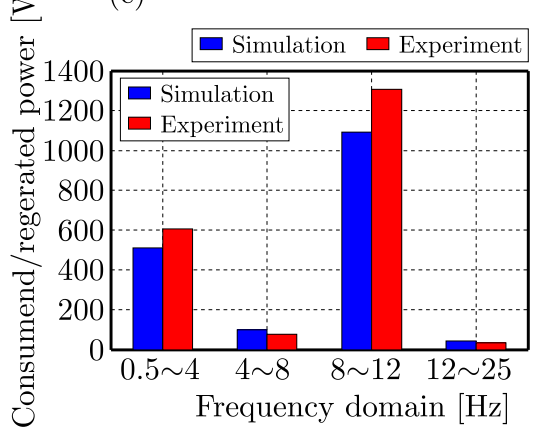

Fig. 13. Comparison between the experiment and simulation: (a) body acceleration, (b) dynamic tire load, (c) consumed /regenerated power

Figure 13 shows a comparison between the test and simulation results. From Figs. 13a and $13 \mathrm{~b}$, it can be seen that there is a certain error between the test and simulation. Specifically, the test results of body acceleration are too large (the error of each frequency band is $18.1 \%$, $20.3 \%, 20.5 \%, 19.8 \%$, respectively) while that of the dynamic wheel load are too small $(6.5 \%$, $7.2 \%, 8.9 \%, 9.1 \%)$. This is mainly because the linear model set in simulation analysis ignores a number of nonlinear factors, such as internal friction of the hydraulic damper and linear motor, asymmetry of the damper damping force and so on. But in the course of the test, the above nonlinear factors are inevitably produced, which makes the "stiffness" increase in the whole 
system. The greater the stiffness, the poorer the riding comfort, and the better the grounding of the tire. For this reason, the body acceleration is increased and the dynamic tire load is reduced. Figure 13c shows the power comparison of energy supply/regeneration. It can be seen from the figure that the energy consumed by the HEMS in the active control of the test is significantly higher than that in the simulation (increased by $18.6 \%$ and $19.7 \%$, respectively), while the energy regenerated in the passive energy feeding mode is contrary to it (reduced by $20.6 \%$ and $19 \%$, respectively). The main reason is that the simulation model only takes the internal resistance of the linear motor into account but ignores the resistance of the control circuit. At the same time, temperature of the motor will be increased during the test process, and it will lead to an increase in energy consumption.

\section{Conclusion}

This paper presents a hybrid electromagnetic actuator as the research object, and designs a multi-mode switching control strategy and its control system. The main conclusions are as follows:

- The hybrid electromagnetic actuator can solve the problem that the electromagnetic suspension with a linear motor alone has poor reliability and cannot guarantee the basic dynamic performance of the system during the process of energy regeneration.

- The HEMS can realize automatic switching between different modes (passive energy regeneration mode, active control comfort mode and active control safety mode) according to the road excitation frequency. In the active control comfort mode, it can simultaneously improve the ride comfort and the tire grounding (compared with PS, it increases by $78 \%$ and $77.2 \%$ respectively). In the active control safety mode, it can improve the tire grounding (compared to PS, it increases by $22 \%$ ) without leading to deterioration of the ride comfort. In the passive energy regeneration mode, a part of the vibrational energy can be regenerated while ensuring the basic dynamic performance. The energy regeneration accounts for $8.75 \%$ of energy consumption in the active control. The simulation results show that the multi-mode switching control of HEMS based on traffic adaptation can effectively balance the dynamic performance and the energy saving.

- There are some errors between the test results and simulation results, this is mainly because the simulation model is linear but there are many nonlinear factors existing in the test process. However, correctness of the simulation results is verified because these errors are within the appropriate range.

- There is a delay and jitter in each mode switching during the experiment, which remains as the main problem to be solved in the following research. At the same time, the multi-mode switching control strategy is based on the regular road input, although it does not have the generality (it is difficult to detect the frequency on a random road). This calls for a new method for mode switching of electromagnetic suspension.

\section{Acknowledgement}

We gratefully acknowledged that this work was financially supported by a grant from National Natural Science Foundation Project, China (project 51970523) and Key Laboratory for New Technology Application of Road Conveyance of Jiangsu Province (project BM20082061710).

\section{References}

1. Asadi E., Ribeiro R., Khamesee M.B., Khajepour A., 2017, Analysis, prototyping, and experimental characterization of an adaptive hybrid electromagnetic damper for automotive suspension systems, IEEE Transactions on Vehicular Technology, 66, 5, 3703-3713 
2. Ataei M., Asadi E., Goodarzi A., Khajepour A., Khamesee M.B., 2017, Multi-objective optimization of a hybrid electromagnetic suspension system for ride comfort, road holding and regenerated power, Journal of Vibration and Control, 23, 5, 782-793

3. Bose suspension system, 2004, Bose Corporation

4. Ding R., Wang R., Meng X., 2018, Energy-saving control strategy design and structure realization for electromagnetic active suspension, Proceedings of the Institution of Mechanical Engineers, Part C: Journal of Mechanical Engineering Science, 233, 9, 3060-3075

5. Ding R., Wang R., Meng X., Chen L., 2017, Study on coordinated control of the energy regeneration and the vibration isolation in a hybrid electromagnetic suspension, Proceedings of the Institution of Mechanical Engineers, Part D: Journal of Automobile Engineering, 231, 11, 1530-1539

6. Ding R., Wang R., Meng X., Chen L., 2019, A modified energy-saving skyhook for active suspension based on a hybrid electromagnetic actuator, Journal of Vibration and Control, 25, 2, 286-297

7. Li Z., Zuo L., Luhrs G., Lin L., Qin Y.-X., 2013, Electromagnetic energy-harvesting shock absorbers: design, modeling, and road tests, IEEE Transactions on Vehicular Technology, 62, 3, 1065-1074

8. Lv C., Zhang J., Li Y., YuAn Y., 2015, Mechanism analysis and evaluation methodology of regenerative braking contribution to energy efficiency improvement of electrified vehicles, Energy Conversion and Management, 92, 469-482

9. Martins I., Esteves M., Pina da Silva F., Verdelho P., 1999, Electromagnetic hybrid active-passive vehicle suspension system, 1999 IEEE 49th Vehicular Technology Conference, 3, 2273-2277

10. Martins I., Esteves J., Marques G.D., Pina Da Silva F., 2006, Permanent-magnets linear actuators applicability in automobile active suspensions, IEEE Transactions on Vehicular Technology, 55, 1, 86-94

11. Shi D., Chen L., Wang R., Jiang H., Shen Y., 2014, Design and experiment study of a semi-active energy-regenerative suspension system, Smart Materials and Structures, 24, 1, 015001

12. Suda Y., Shimba T., Hio K., Kawamoto Y., Kondo T., Yamagata H., 2004, Study on electromagnetic damper for automobiles with nonlinear damping force characteristics (road test and theoretical analysis), Vehicle System Dynamics, 41, 637-646

13. Sun X., Cai Y., Yuan C., Chen L., Wang R., 2017a, Hybrid model predictive control of damping multi-mode switching damper for vehicle suspensions, Journal of Vibroengineering, 19, 4, 2910-2930

14. Sun X., Yuan C., Cai Y., Wang S., Chen L., 2017b, Model predictive control of an air suspension system with damping multi-mode switching damper based on hybrid model, Mechanical Systems and Signal Processing, 94, C, 94-110

15. Xie X.D., Wang Q., 2015, Energy harvesting from a vehicle suspension system, Energy, 86, 385-392

16. Wang J., Jewell G.W., Howe D., 2001, Design optimization and comparison of tubular permanent magnet machine topologies, IEE Proceedings-Electric Power Applications, 148, 5, 456-464

17. Wang J., Wang W., Atallah K., 2011, A linear permanent-magnet motor for active vehicle suspension, IEEE Transactions on Vehicular Technology, 60, 1, 55-63

18. Wang R., Ding R., Chen L., 2018, Application of hybrid electromagnetic suspension in vibration energy regeneration and active control, Journal of Vibration and Control, 24, 1, 223-233

19. Zuo L., Chen X., NAYfen S., 2011, Design and analysis of a new type of electromagnetic damper with increased energy density, Journal of Vibration and Acoustics, 133, 4, 041006 